\title{
C2 prosthesis: anterior upper cervical fixation device to reconstruct the second cervical vertebra
}

\author{
Dezső Jeszenszky • Tamás Fülöp Fekete • \\ Robert Melcher · Jürgen Harms
}

Received: 22 April 2007 / Accepted: 24 June 2007/Published online: 14 July 2007

(C) Springer-Verlag 2007

\begin{abstract}
Destruction of the second cervical vertebra leads to a highly unstable situation. Reconstruction is difficult because the axis plays a central role in rotatory movements and has a unique function in redistributing axial loads. The axis transfers the axial load of the two lateral masses of the atlas to three surfaces on the third cervical vertebra: the two articular facets and the vertebral body. As reconstruction is difficult and the instability in this region is life threatening, pathological processes are often treated less radically compared to other areas of the cervical spine. However, this more moderate approach may result in worse outcomes and prognoses. This paper presents the development of a new implant ( $\mathrm{C} 2$ prosthesis) and two illustrative cases describing the implementation of this new implant. The $\mathrm{C} 2$ prosthesis provides anterior support and therefore allows a more radical surgical approach.
\end{abstract}

Keywords Axis neoplasm - Spondylectomyvertebrectomy - Spinal instability $\cdot \mathrm{C} 2$ prosthesis . Anterior support

D. Jeszenszky $(\bowtie) \cdot T$. F. Fekete

Schulthess Clinic, Spine Center, Lengghalde 2,

8008 Zurich, Switzerland

e-mail: dezsoe.jeszenszky@kws.ch

R. Melcher · J. Harms

SRH Klinikum Karlsbad-Langensteinbach,

Center for Spine Surgery, Guttmannstrasse 1,

76307 Karlsbad, Germany

\section{Introduction}

Destruction of the body of the second cervical vertebra may develop due to primary or secondary bone tumours or infections. The treatment of these pathological processes often requires surgical resection, which may further increase instability [14]. Current treatment strategy consists of tumour removal, decompression and posterior fixation $[2-4,8$, $9,13,15]$. If the vertebral body of the axis-including the superior facet joints-is not present either by the destruction of a pathological process or by surgical removal, posterior fixation is widely extended. The usual extent of posterior fixation is from the occiput down to the lower subaxial spine and external immobilization is also applied $[3,6,9,13]$. This fixation has to exceed the biomechanically compromised segments because the missing anterior support has to be compensated for. Some surgeons also perform anterior reconstructions with bone grafts and screws or bone grafts, plates and screws. However, such anterior reconstruction alone is insufficient, the posterior occipito-cervical fixation and external immobilisation cannot be avoided. These combined anterior and posterior approaches are limited to isolated case reports or small series $[1,8,10,11]$. The low numbers of anterior reconstructions might be due to the difficulty in achieving an adequate connection between the $\mathrm{C} 1$ lateral masses located laterally and the $\mathrm{C} 3$ vertebral body in the midline.

To meet the special anatomical and biomechanical requirements of reconstructing the axis, the first and senior authors designed a $\mathrm{C} 2$ prosthesis by integrating the Harms anterior transoral plate and titanium mesh cage. An implant, that restores stability following total removal of the second cervical vertebra, without the need for fixation of the atlantooccipital joints, has, to the authors' knowledge, not been described before. 


\section{Materials and methods}

During surgical interventions of $\mathrm{C} 2$ resection the implants used to reconstruct the axis were serially modified. As the first step of development a transoral T-shaped plate and a Harms cage was assembled prior to each surgery up to 1997 (Fig. 1a-d, g). Later a shorter and wider titanium cage to provide support for the $\mathrm{C} 1$ lateral masses was added (Fig. 1e, h). The prosthesis was custom-made for each patient. The size of the implant was based on measurements of preoperative radiological data. However, later it turned out that two different sizes (large and small) were sufficient for all patients. Therefore the design was finally changed to accommodate for both sizes by intraoperatively shortening the larger size implant when necessary (Fig. 1f, i).

\section{Illustrative case report 1}

A 16 year-old patient had a history of neck pain radiating to the occipital region. MRI scans showed a tumour mass at the level of $\mathrm{C} 1$ and $\mathrm{C} 2$. Suboccipital craniectomy, $\mathrm{C} 1$ and C2 laminectomy, and a partial tumour resection were performed through a posterior transdural approach in another hospital (Fig. 2). The histology revealed chordoma. The patient had long tract signs and lower limb ataxia

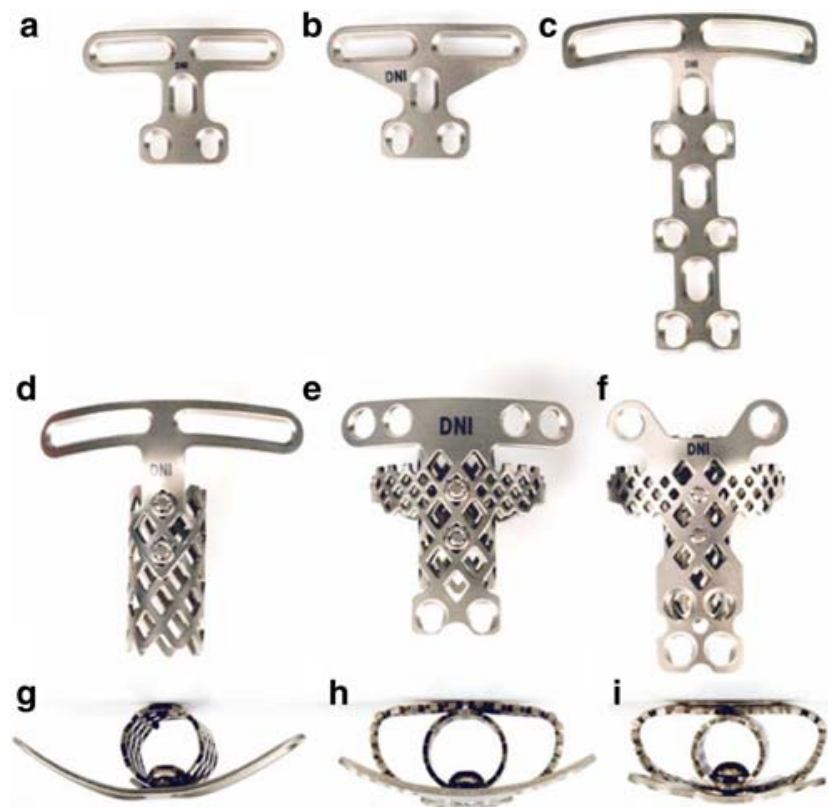

Fig. 1 Evolution of $\mathrm{C} 2$ prosthesis. a-c Original transoral T-shaped plate. d Integration of transoral plate with a titanium mesh cage. e A wider elliptical cylinder cage was added to provide support for the lateral mass of atlas. $\mathbf{f}$ final version. The horizontal part of the plate was shortened to ease insertion and the caudal end of the implant was changed to provide longitudinal adaptability. $\mathbf{g}-\mathbf{i}$ Corresponding superior view of the implants from $\mathbf{d}$ to $\mathbf{f}$

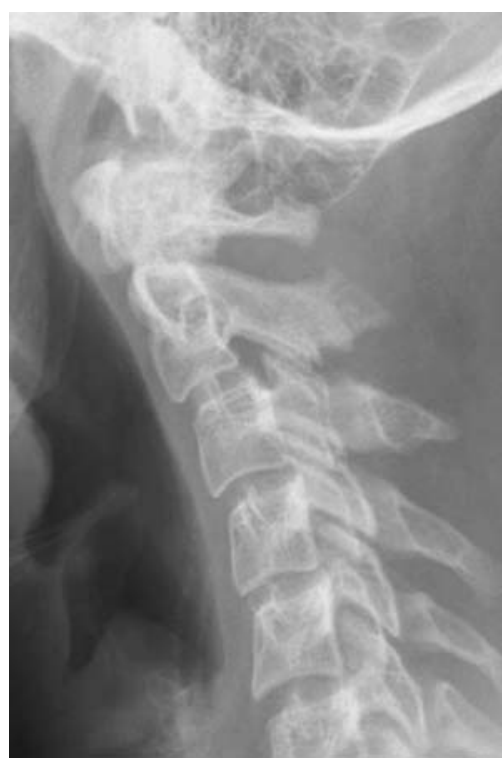

Fig. 2 Signs of $\mathrm{C} 1$ and $\mathrm{C} 2$ laminectomy and kyphotic angulation are visible on the lateral cervical spine $\mathrm{X}$-ray

at the postoperative period, which spontaneously resolved in a few weeks. The patient was referred to our hospital for complete tumour removal before proton beam therapy.

We removed the $\mathrm{C} 2$ vertebral body through a high cervical left anterolateral approach. Following total tumour removal it was evident that the atlas was not involved, the lateral masses and anterior arch were intact. We then implanted the $\mathrm{C} 2$ prosthesis. The $\mathrm{C} 1$ lateral mass screws were inserted through two stab incisions on the pharynx in a transoral approach. We turned the patient and performed a posterior $\mathrm{C} 1-\mathrm{C} 3$ fixation using polyaxial screws and rods.

The patient was mobilised without any orthosis and the recovery period was uneventful. Postoperative radiographs showed well-situated implants (Fig. 3). There was no evidence of residual tumour on the postoperative MRI.

Illustrative case report 2

A 51 year-old patient suffered from progressive neck pain. Radiological investigation revealed a left-sided tumour partially destroying the $\mathrm{C} 2$ and $\mathrm{C} 3$ vertebral bodies. Posterior decompression, C2, C3, C4 laminectomy and tumour resection was performed in another institution. The result of the histological analysis was chordoma.

The patient was referred to our hospital to complete the removal of the tumour before a proton beam irradiation.

We performed a posterior fusion from $\mathrm{C} 1$ to $\mathrm{C} 5$, using polyaxial lateral mass screws and rods, and restored a lordotic curve. We decided to include the $\mathrm{C} 4-5$ segment in the stabilisation, because of significant instability from previous surgery. At that stage we turned the patient and 

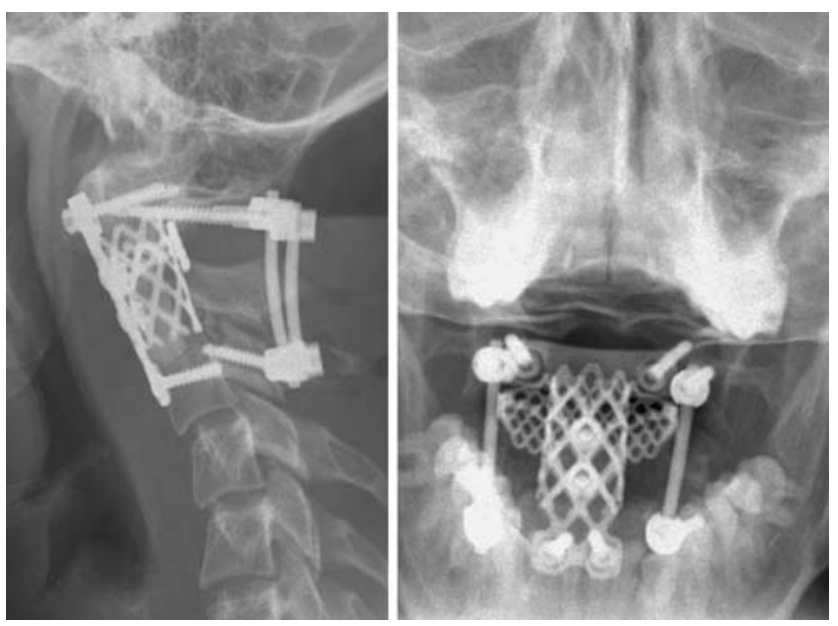

Fig. 3 Postoperative radiographs show good positioning of the C2 prosthesis, the posterior screws and rods. The atlanto-occipital segment was spared. The preoperatively seen kyphotic angulation below the level of $\mathrm{C} 3$ was unchanged

performed total tumour removal, an anterior C2, C3 vertebral body resection and a left-sided vertebral artery resection. We also performed a $\mathrm{C} 4-5$ discectomy and fusion. A custom-made $\mathrm{C} 2$ prosthesis was used to reconstruct the anterior aspect of the spine. The length of the cylindrical cage was long enough to replace both the $\mathrm{C} 2$ and $\mathrm{C} 3$ vertebral bodies. The right third of the $\mathrm{C} 2$ vertebral body seemed to be intact and preservation was possible. The wing of the $\mathrm{C} 2$ prosthesis on the right side was removed accordingly (Fig. 4). The anterior plate of the prosthesis was made especially long to incorporate the C5 vertebra into the fusion. The third approach in the same session was transoral. Two screws to the lateral mass of $\mathrm{C} 1$ were

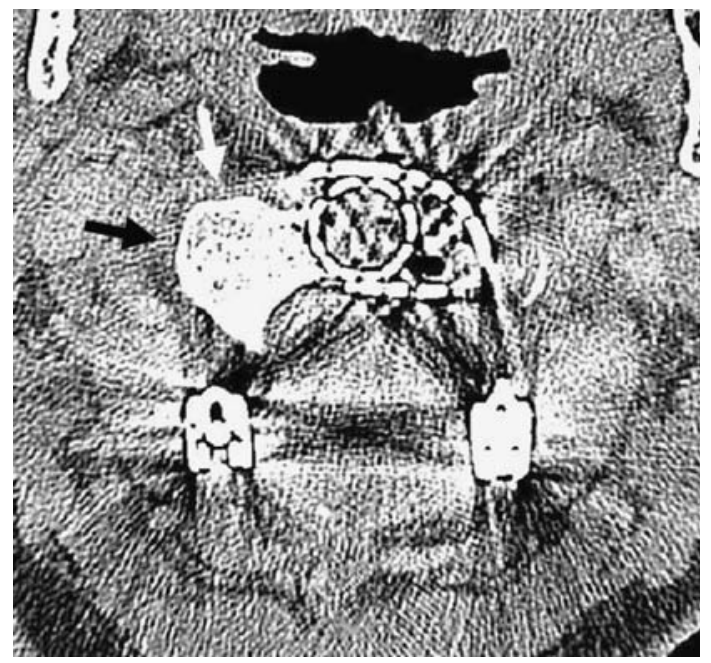

Fig. 4 Axial CT image at the $\mathrm{C} 2$ level. The right half of the $\mathrm{C} 2$ body was spared (arrows), so the right wing of the prosthesis was removed accordingly to adapt to the situation inserted through two stab incisions on the posterior wall of the pharynx to stabilise the cranial end of the T-shaped plate of the $\mathrm{C} 2$ prosthesis.

The patient was extubated on the third postoperative day and mobilised without any external immobilisation device. Unfortunately, there was a haematoma formation at the anterior surgical site so the anterior wound on the tenth postoperative day was revised and the haematoma was evacuated. There was no implant failure and there was no neurological complication. The postoperative radiographs showed a good alignment of the cervical spine (Fig. 5).

Later the patient received proton beam therapy. At 2-year follow-up the patient is still tumour-free. The patient is fully reintegrated into normal daily activities.

\section{Results: description of $\mathrm{C} 2$ prosthesis}

The prosthesis consists of a T-shaped plate, a long cylinder mesh cage, and a short elliptical cylinder mesh cage (Fig. 1f, i). The width of the elliptical cylinder cage is $25 \mathrm{~mm}$, the depth is $14 \mathrm{~mm}$ and the height is $10 \mathrm{~mm}$. The diameter of the cylinder cage is $14 \mathrm{~mm}$. The elliptical cylinder cage is situated at the cranial end of the main cylinder cage. The horizontal part of the T-shaped plate is just above the main cylinder cage and the caudal part of the plate extends below the main cylinder cage. All three components are built together as a robust construct.

The elliptical cylinder cage provides axial support to the inferior articular facets of the atlas. The load of the elliptical cylinder cage is transferred to the caudally adjacent vertebra via the main cylinder cage (Fig. 8).
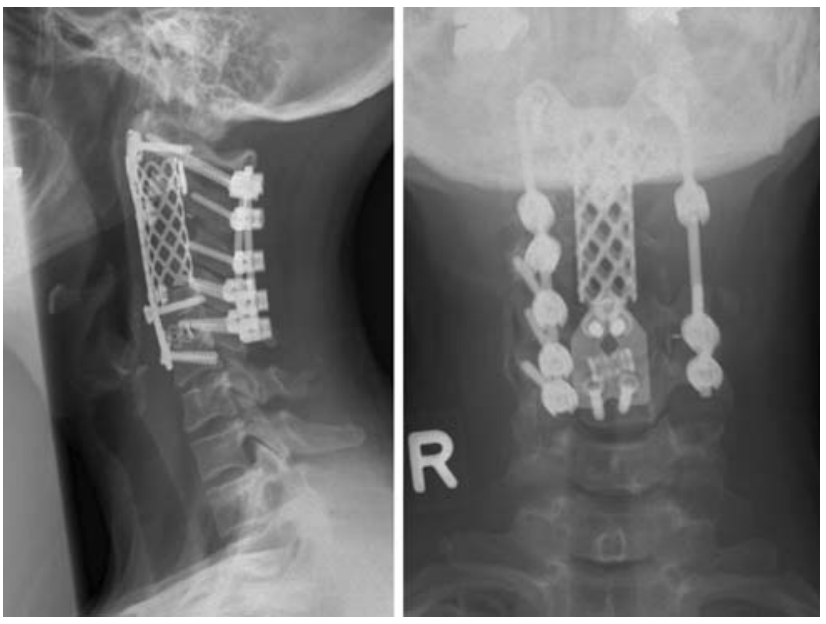

Fig. 5 Postoperative lateral an AP radiographs showing the prosthesis replacing the partially and completely removed $\mathrm{C} 2$ and $\mathrm{C} 3$ vertebral bodies, respectively 


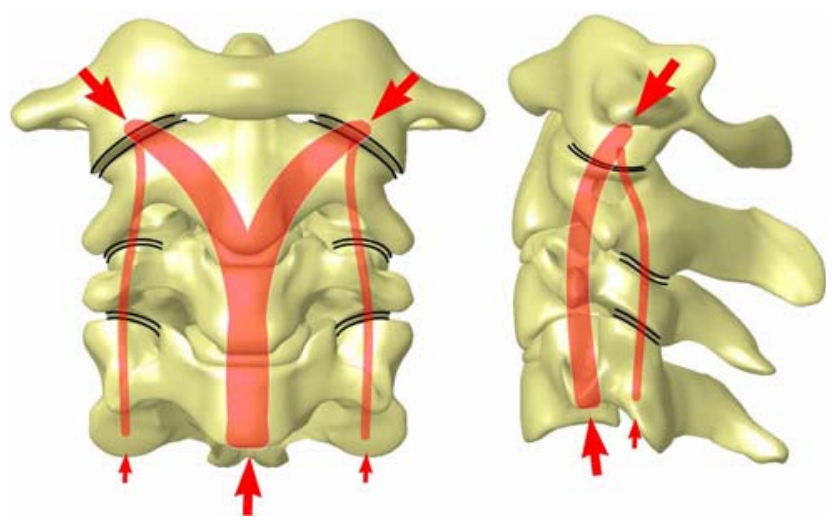

Fig. 6 This figure illustrates the axial load redistribution of the atlas: the 2-column system in the atlantoaxial complex and 3-column system in the $\mathrm{C} 2-\mathrm{C} 3$ complex. The transparent red lines indicate the axial forces through the upper cervical spine. The width of the lines corresponds to the magnitude of force. The short black lines indicate contours of the articular facets
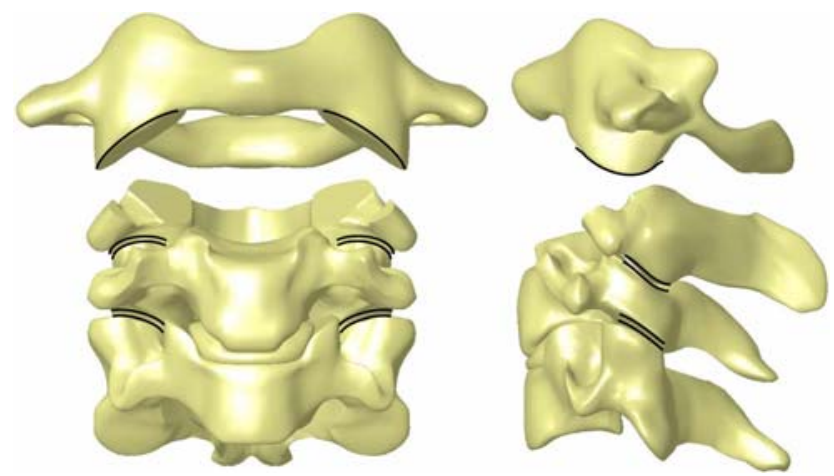

Fig. 7 This figure illustrates how $\mathrm{C} 2$ body resection leads to complete disconnection of the spine. The body of the axis vertebra, along with the dens and superior articular facets, were removed
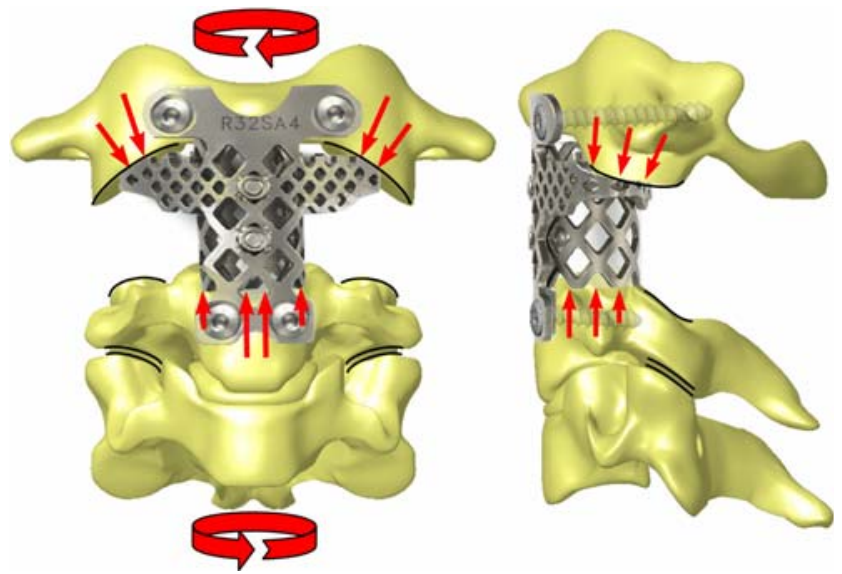

Fig. 8 This figure illustrates the anterior support and rotational stability provided by the $\mathrm{C} 2$ prosthesis
There is one hole on each side at the horizontal part of the plate for screws to the lateral mass of $\mathrm{C} 1$. There are four holes in two rows at the caudal end of the plate for two screws to the next adjacent subaxial vertebral body. The screw holes in the plate are shaped in a compressive fashion, i.e. when tightening the screws the prosthesis is compressed between the lateral mass of the atlas and the caudally adjacent subaxial vertebra. The holes are designed for bicortical screws with a diameter of 3.5 or $4 \mathrm{~mm}$.

A combined anterolateral and transoral surgical approach was used in both cases described here, however, a transoral approach described by Schmelzle and Harms [12] is generally used.

Transition of the large $\mathrm{C} 2$ prosthesis to the small size

There are four holes at the caudal end of the plate, as mentioned above. By cutting off the distal end of the plate with the two holes and, accordingly, cutting off the caudal end of the mesh cage, the implant can be used where the smaller size is needed. Furthermore, the prosthesis can be individually modified intraoperatively just like the Harms mesh cage. The shape of implant can be modified according to the type and extent of resection.

The length of the main cylinder cage can depend on the extent of resection. In individual cases an implant can be made to reconstruct a longer segment of the cervical spine with an appropriately long main cylinder cage and plate. This allows for the resection of additional cervical vertebral bodies. The extent of resection this implant still provides a safe support for is still unknown.

\section{Discussion}

\section{Anatomical and biomechanical considerations}

The craniovertebral junction is the most mobile portion of the spinal column accounting for about half of all movements in the cervical spine. The second cervical vertebra is a transition between the atlas and the more typical third cervical vertebra. The role of axis in the rotatory movement of the atlantoaxial complex is well known, as its name implies. However, the unique arrangement of its articular facets, along with its special role in the redistribution of axial load, is rarely discussed.

Unlike any other vertebra, the superior articular facets of the axis originate from the vertebral body and extend posteriorly onto the adjoining parts of the pedicles. The inferior articular facets are situated at the junction of the pedicles and laminae. Thus the superior articular facets are anteriorly situated with respect to the inferior articular facets. The consequences are twofold. 
First, the superior facets do not form an articular pillar with the inferior facets. As $\mathrm{C} 1$ lacks a vertebral body, the axial load of atlas is transferred through the C1-C2 facet joints on both sides and can be regarded as a two-column (left and right) system. The joints between the axis and the third cervical vertebra are the $\mathrm{C} 2-\mathrm{C} 3$ facet joints and the $\mathrm{C} 2-\mathrm{C} 3$ intervertebral disc. Thus, the $\mathrm{C} 2-\mathrm{C} 3$ joints represent a three-column system. The unique role of the body of axis is to redistribute the two-column axial load of the atlas into a three-column system of the subaxial spine (Fig. 6).

Second, the removal of the body of the axis includes removal of the superior articular facets, thereby separating all the connections of the atlas to the pedicle of the axis and thus to the inferior articular facets of $\mathrm{C} 2$. This is practically a iatrogenic lesion in the pars interarticaluris of the axis. Vertebral body resection at C2 level through a single anterior approach results in complete disconnection of the spine (Fig. 7)! This is equivalent to a $360^{\circ}$ surgical decompression/resection at any other lower spinal level. Therefore following removal of the body of the axis, both anterior and posterior structures have to be reconstructed.

\section{Clinical implications}

Anterior reconstruction following axis removal is difficult with the usual strut grafts. The caudal end of the graft material can be secured to the subaxial vertebral body just like anywhere else in the subaxial spine. However, to establish a secure connection at the cranial end of the conventional implant to the atlas is more difficult. The cranial end of a conventional cage or the bone graft can be secured either by an anterior plate or direct screws to the tiny anterior arch of the atlas [11]. However, neither of these methods provide safe fixation. The anterior support is not reliable, because the bone graft or the cage with a relatively small diameter can only support the thin anterior arch of the atlas and not the more important lateral masses. In addition to this the screw purchase in the arch of the atlas is also very poor.

As a result the benefit of adding such an anterior support is at least questionable. This is supported by the authors' experience and by the small number of reported cases where the fixation failed and/or extensive posterior fixation and external immobilisation was used $[11,15]$.

Another difficult area following removal of the axis is the counteraction of active rotational forces as muscles acting to rotate the head still remain intact.

In contrast, the $\mathrm{C} 2$ prosthesis provides a reliable anterior support and rotational stability. The implant presented in this paper was designed to replace the above-mentioned roles of the axis in axial load redistribution.
Application of the $\mathrm{C} 2$ prosthesis

The $\mathrm{C} 2$ prosthesis provides anterior support to the atlas by the wide elliptical cylinder cage (wings of the prosthesis), which transfers the axial loads of the $\mathrm{C} 1$ lateral masses to the vertebral body of the subaxial vertebra in the midline (Fig. 8). In addition, the prosthesis also provides rotational stability as it is secured to the lateral masses of the atlas by screws through the wide upper portion of the T-shaped plate.

The corrugated ends of the elliptical cylinder cage are designed to embed themselves at $\mathrm{C} 1$ lateral masses and at the most cranially available subaxial vertebra to prevent any translational or rotational movement. The plate prevents migration of the implant toward the spinal canal.

As mentioned earlier, an additional posterior fixation is unavoidable since the removal of the body of the axis completely disconnects the spine. This is also supported by a biomechanical study, where additional posterior stabilisation improved stability of transoral atlantoaxial plating [7]. The authors' method of choice for a posterior fixation is polyaxial screws and rods. However, the extent of the posterior fixation can be reduced in comparison to previously described methods. In contrast to formerly known methods of anterior support, a stable connection is provided between the atlas and the anterior construct. There are four screws in the atlas with the anterior and posterior lateral mass screws. Anterior support is provided by the wings of the implant supporting the lateral masses. As a consequence, an occipitocervical fixation is unnecessary; the motion of $\mathrm{C} 0-\mathrm{C} 1$ segment can be preserved. It also allows a more moderate approach to the rest of the subaxial spine, as the posterior fixation can caudally end at the first available unaffected subaxial vertebra.

The combined anterior $\mathrm{C} 2$ prosthesis and posterior screw-rod construct achieves immediate stability. The safe reconstruction allows a more radical approach to pathologies affecting the axis. Thus the generally accepted concepts in spine surgery can be applied in cases of destruction of the axis vertebra. This might result in improved clinical outcome, longer survival, and higher rate of curative surgical interventions.

The decreased extent of fixation preserves unaffected motion segments. Furthermore, the use of an external orthosis is not necessary. All these improve the quality of life.

To the authors' knowledge, there has been no previously published description of total axis removal without immobilizing the $\mathrm{C} 0-1$ segment to date.

Initial results with the $\mathrm{C} 2$ prosthesis proved to be excellent and without hardware failure [5]. 


\section{Conclusions}

The new prosthesis was constructed to replace the second cervical vertebra. This is the first implant which allows complete removal of $\mathrm{C} 2$ while preserving $\mathrm{C} 0-\mathrm{C} 1$ motion. The combined anterior $\mathrm{C} 2$ prosthesis and posterior screwrod construct achieves immediate stability, obviating the need for an external orthosis and allowing for early mobilisation.

The new implant allows a more radical surgical approach to spinal pathologies involving the axis vertebra. The implant can also be applied in a clinical setting, where the pathological process exceeds the confines of the axis in the caudal direction and destroys subaxial vertebrae. The C2 prosthesis allows the application of concepts generally accepted in other areas of spine surgery.

The benefit of this new fixation method is currently being investigated in a clinical study with 10 -year followup at the time of writing.

Acknowledgments The authors would like to thank BiedermannMotech $\mathrm{GmbH}$ for construction and development of the $\mathrm{C} 2$ prosthesis; Wilfried Matthis for all the support in biomechanics and the preparations of the 3D illustrations; Andreas Lütscher for the photographs; and Charles McCammon for helping to prepare the manuscript.

\section{References}

1. Bailey CS, Fisher CG, Boyd MC, Dvorak MF (2006) En bloc marginal excision of a multilevel cervical chordoma. Case report. J Neurosurg Spine 4:409-414

2. Dickman CA, Locantro J, Fessler RG (1992) The influence of transoral odontoid resection on stability of the craniovertebral junction. J Neurosurg 77:525-530
3. Fourney DR, York JE, Cohen ZR, Suki D, Rhines LD, Gokaslan ZL (2003) Management of atlantoaxial metastases with posterior occipitocervical stabilization. J Neurosurg 98:165-170

4. Hertlein H, Mittlmeier T, Schurmann M, Lob G (1994) Posterior stabilization of $\mathrm{C} 2$ metastases by combination of atlantoaxial screw fixation and hook plate. Eur Spine J 3:52-55

5. Jeszenszky D, Harms J, Hadasch R, Preiss S (1999) C2 prosthesis allowing optimal stabilisation after $\mathrm{C} 2$ resection following destructive lesions. Eur Spine J 8:S40, P38

6. Jonsson B, Jonsson H Jr., Karlstrom G, Sjostrom L (1994) Surgery of cervical spine metastases: a retrospective study. Eur Spine J 3:76-83

7. Kandziora F, Kerschbaumer F, Starker M, Mittlmeier T (2000) Biomechanical assessment of transoral plate fixation for atlantoaxial instability. Spine 25:1555-1561

8. Levin DA, Hensinger RN, Graziano GP (2006) Aneurysmal bone cyst of the second cervical vertebrae causing multilevel upper cervical instability. J Spinal Disord Tech 19:73-75

9. Piper JG, Menezes AH (1996) Management strategies for tumors of the axis vertebra. J Neurosurg 84:543-551

10. Rao G, Ha CS, Chakrabarti I, Feiz-Erfan I, Mendel E, Rhines LD (2006) Multiple myeloma of the cervical spine: treatment strategies for pain and spinal instability. J Neurosurg Spine 5:140-145

11. Rhines LD, Fourney DR, Siadati A, Suk I, Gokaslan ZL (2005) En bloc resection of multilevel cervical chordoma with C-2 involvement. Case report and description of operative technique. J Neurosurg Spine 2:199-205

12. Schmelzle R, Harms J (1987) Craniocervical junction-diseases, diagnostic application of imaging procedures, surgical techniques. Fortschr Kiefer Gesichtschir 32:206-208

13. Shin H, Barrenechea IJ, Lesser J, Sen C, Perin NI (2006) Occipitocervical fusion after resection of craniovertebral junction tumors. J Neurosurg Spine 4:137-144

14. Stoltze D, Harms J (1991) Cervico-occipitale Stabilisierungen unter besonderer Berücksichtigung der transoralen Technik. Georg Thieme Verlag, Stuttgart

15. Tuite GF, Veres R, Crockard HA, Sell D (1996) Pediatric transoral surgery: indications, complications, and long-term outcome. J Neurosurg 84:573-583 